\title{
Antipodal Vivaldi Antenna for Water Pipe Sensor and Telemetry
}

\author{
Giuseppe Ruvio, Domenico Gaetano, Max J. Ammann, and Patrick McEvoy \\ Antenna \& High Frequency Research Centre, Dublin Institute of Technology, Kevin Street, Dublin 8, Ireland \\ Correspondence should be addressed to Giuseppe Ruvio, giuseppe.ruvio@dit.ie
}

Received 20 March 2012; Accepted 5 May 2012

Academic Editor: Francesco Soldovieri

Copyright ( 2012 Giuseppe Ruvio et al. This is an open access article distributed under the Creative Commons Attribution License, which permits unrestricted use, distribution, and reproduction in any medium, provided the original work is properly cited.

\begin{abstract}
An antipodal Vivaldi antenna operates simultaneously for telemetry and sensing when placed conformally onto PVC pipes. Good radiation efficiency is realised and the antenna impedance matching remains stable when a pipe is empty or contains water. The fidelity factor performance based on an input Ultra-Wideband Gaussian pulse is a suitable figure of merit to detect water presence. Different water levels and pipe conditions correspond to fidelity factors between $90 \%$ and $96 \%$, which are a suitable dynamic range for sensing and the appropriate quality for pulse communications for remote monitoring.
\end{abstract}

\section{Introduction}

Monitoring pipe systems represents a major problem where continuous information on the liquid content must be constantly provided. This is the case for water leakage sensor networks or for stormwater detection in critical drainage system. Several technologies were proposed in the literature for these applications such as tracer gases and thermography [1]. Furthermore, ultrasound sensors have been proposed for accurate detection and localisation of leakages in buried water pipes [2,3]. Acoustic wave monitoring is widely used in leak detection but they are concerns in terms of time response, robustness, reliability, accuracy, and costs. Radiofrequency telemetry has been more recently introduced to localize leaks and malfunctioning pipelines. In particular, Ground Penetrating Radar (GPR) systems can be efficiently designed to image subsurface scattering leaks by using lowfrequency electromagnetic (EM) waves $[4,5]$. However, due to their cost and characteristics, GPR is suitable for extraordinary maintenance. EM sensors revealing wave propagation distortion inside metallic pipes have been recently proposed [6]. Although reflections coming from leaks/cracks can be promptly identified by EM propagating mode monitoring, this technique is restricted to a limited number of applications and scenarios.

High data rates and low-power requirements make UltraWideband (UWB) technology largely used for short-range wireless communication and sensor systems. With a large operating spectrum, short pulses can be generated. UWB sensors can then monitor pulse distortion and identify the associated environmental changes. Operating in the time domain implies the design of novel figures of merit to characterize UWB impulse radio systems. The signal purity in particular can be used to assess the time domain performance of the UWB front-end for antenna and sensor operative scenarios. This can be measured in terms of fidelity factor, which estimates the distortion of the received signal with respect to the original transmitted signal. This paper introduces a conformal antipodal Vivaldi antenna operating in the UWB range to be placed on Polyvinyl Chloride (PVC) pipes. This UWB directional antenna has been designed to fit onto a curved surface of a circular cross-section pipe for communication between flow rate meter devices [7]. The use of wireless monitoring technology to aid fluid flow control can improve reliability, reduce costs, and save space. This was proposed in [8] where an acoustic sensor is combined with to a radiotransmitter which communicates with an external gateway.

In this paper, an innovative UWB antenna for both sensing and data transmission is proposed for pipe telemetry applications. A preliminary study of the pipe proximity effects on a directional UWB antenna in the frequency domain [9] is extended by a thorough time domain analysis. Four standard PVC pipes have been numerically evaluated 


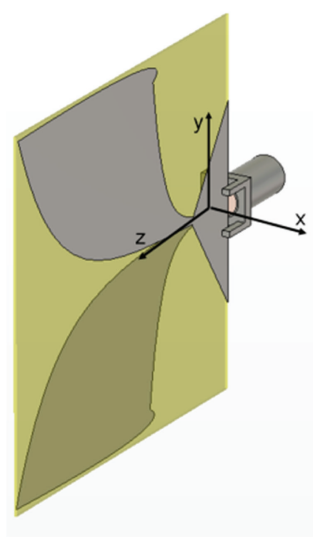

(a)

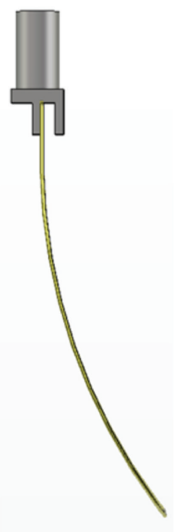

(b)

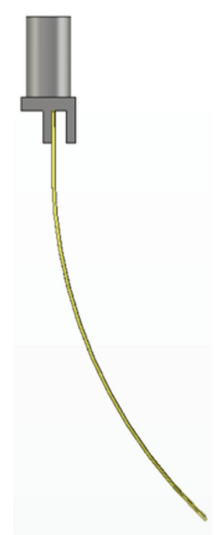

(c)

FIGURE 1: Antipodal Vivaldi antenna geometry and coordinate system; (a) flat configuration; (b) bent on 141.2 mm diameter of curvature; bent on $114.3 \mathrm{~mm}$ diameter of curvature.

for different water content levels using the finite integration time domain technique (CST Microwave Studio [10]). Numerical results show that the conformal antipodal Vivaldi antenna is suitable for sensing the water level inside the pipe and for simultaneous transmission to an external metering station. These combined operations lead to significant simplification and cost saving for the metering system. In particular, the fidelity factor is shown to be a convenient metric to sense changes in water presence and to assess radiation properties. The time domain performance of the system is assessed by calculating the fidelity of the received pulse at a number of probes circularly distributed around the antenna in the $\mathrm{H}$-plane with a $5^{\circ}$ step. The quality of a received pulse through the antenna system and the RF channel can be evaluated by using the following expression [11]:

$$
\mathrm{FF}=\max _{\tau} \int_{-\infty}^{\infty} L\left[\mathrm{p}_{\text {source }}(t)\right] \mathrm{p}_{\text {output }}(t-\tau) d t
$$

where the source pulse $\mathrm{p}_{\text {source }}(t)$ and output pulse $\mathrm{p}_{\text {output }}(t)$ are normalised by their energy, respectively. The fidelity factor FF is the maximum integration by varying time delay $\tau$. The linear operator $L[\cdot]$ describes the system transformation occurring in the input signal $\mathrm{p}_{\text {source }}(t)$. In this case, as the transmit antenna is a Vivaldi type, a differentiation of the source pulse occurs [12] and the operator $L[\cdot]$ corresponds to $d[\cdot] / d t$.

The operating scenario is envisioned as follows. The pipe mounted antenna/sensor communicates to a UWB IEEE 802.15.4 node which can also be integrated in a WiFi access point (AP). The UWB range is dependent on the data bandwidth, which in this case can be quite small, allowing a range of tens of meters. The position of antennas on the pipe should be considered in relation to the AP location.

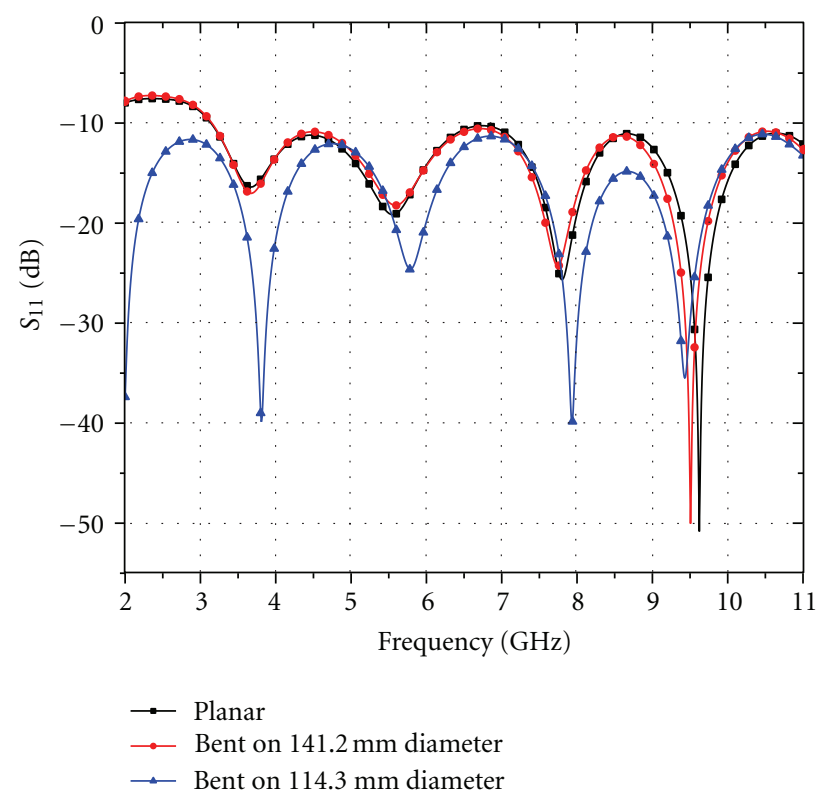

Figure 2: $S_{11}$ of free-space planar and conformal antenna.

\section{Antipodal Vivaldi Antenna and Testing Environment}

An antipodal Vivaldi antenna was designed to operate across the FCC UWB spectrum $(3.1-10.6 \mathrm{GHz})$ when mounted on a pipe surface. The antenna is double-side printed on $0.375 \mathrm{~mm}$ thin Duroid 5870 dielectric substrate $\left(\varepsilon_{r}=\right.$ 2.33 , loss tangent $=0.0012$ ) with overall dimensions $50 \times 53 \mathrm{~mm}$. This dielectric material was chosen for its stable electrical properties combined with mechanical flexibility that permits the antenna to be curved on a surface of a cylindrical object. Figure 1(a) shows the antenna geometry and the coordinate system. The tapered antenna aperture generates multiple resonances resulting in 

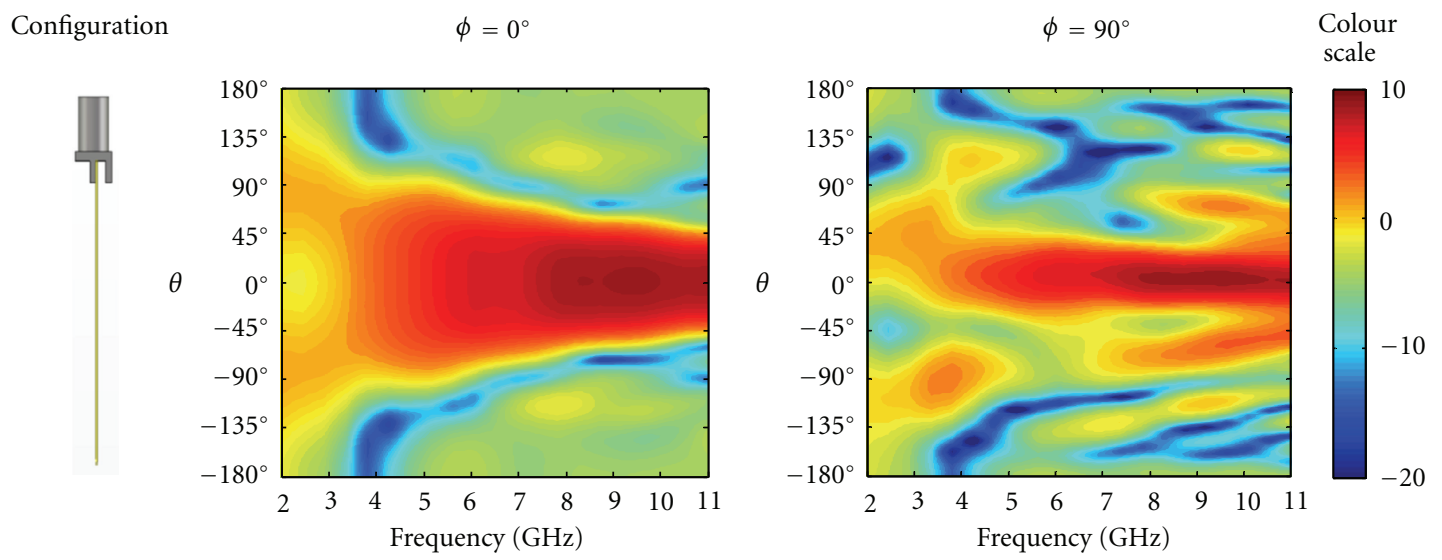

(a)
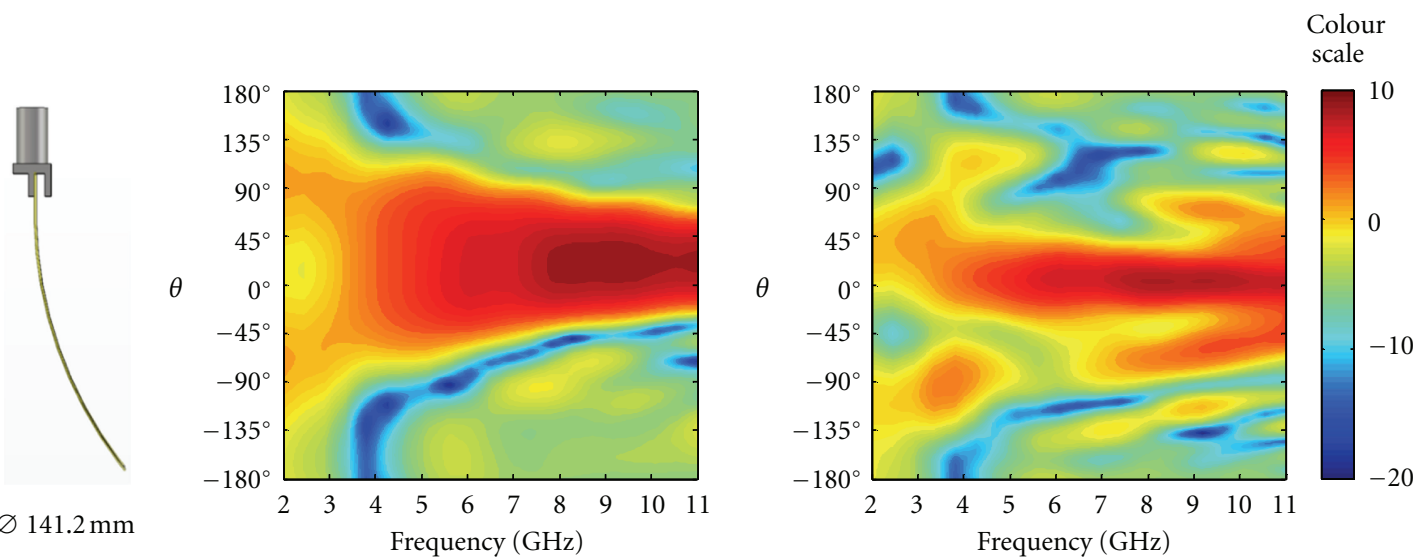

(b)

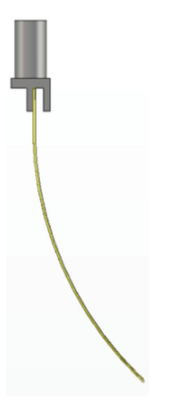

$\varnothing 114.3 \mathrm{~mm}$


(c)

Figure 3: 2-D radiation patterns ( $\mathrm{dBi}$ ) of (a) the planar antenna in free space for $\phi=0^{\circ}$ (first column) and $\phi=90^{\circ}$ (second column); (b) the conformal antenna to a $141.2 \mathrm{~mm}$ diameter of curvature in free space for $\phi=0^{\circ}$ and $\phi=90^{\circ}$; (c) the conformal antenna to a $114.3 \mathrm{~mm}$ diameter of curvature in free space for $\phi=0^{\circ}$ and $\phi=90^{\circ}$.

a continuous operating UWB bandwidth and a uniform endfire directed radiation pattern. Figures 1 (b) and 1(c) show the antenna curvature when the longitudinal axis of the pipe is rotated orthogonally to the antenna end-fire direction on $141.2 \mathrm{~mm}$ and $114.3 \mathrm{~mm}$ diameters of curvature, respectively.
In particular, the tapered line of the flare shape is described by two exponential curves given by (2) and then mirrored on the opposite side of the dielectric:

$$
\begin{array}{cc}
y=0.125 * e^{0.109 z} & \text { for } 0<z<48.5[\mathrm{~mm}], \\
y=0.11 * e^{0.352 z} & \text { for } 0<z<15[\mathrm{~mm}]
\end{array}
$$


TABle 1: Pipe configurations investigated.

\begin{tabular}{lcc}
\hline Pipe configuration & Diameter $[\mathrm{mm}]$ & Thickness $[\mathrm{mm}]$ \\
\hline A & 114.3 & 6.02 \\
B & 114.3 & 8.56 \\
C & 141.2 & 6.55 \\
D & 141.2 & 9.50 \\
\hline
\end{tabular}

for the internal and external tapered profile of each flare, respectively.

In order to reduce the back lobe radiation, the exponential lines were connected with spline shapes [13]. Finally, a tapered microstrip feeding line was designed to secure a broadband $50 \Omega$ impedance condition in the intersection point [14].

2.1. Free-Space Antenna Characteristics. The performance of this antipodal Vivaldi antenna shows it to be resilient to mechanical bending along the $y$-axis. Figure 2 shows the free-space $S_{11}$ of the antenna in its flat and both curved configurations. The impedance matching expressed in terms of $S_{11}$ remains stable across all configurations and below $-10 \mathrm{~dB}$ over the complete UWB bandwidth.

Three-dimensional broadband free-space radiation patterns of the antenna in its flat and both curved configurations are shown in Figure 3. The patterns exhibit the same shape and high gain in the end-fire direction. Due to the antipodal configuration the beam in the $\phi=90^{\circ}$ direction is not totally symmetrical. Bending the antenna affects the radiation pattern in its $\phi=0^{\circ}$ cut as this corresponds to a rotation of the tapered line termination. As expected, by decreasing the diameter of curvature, the direction of maximum radiation moves from the $\theta=0^{\circ}$ direction towards the angular range $20^{\circ}-30^{\circ}$. Moreover, significant radiation pattern stability can be observed across the entire FCC UWB band.

2.2. Pipe Characterization. Four different ASTM standard PVC pipes $\left(\varepsilon_{r}=2.9\right.$; loss tangent $=0.086$ at $f=2 \mathrm{GHz}$ and 0.0182 at $f=11 \mathrm{GHz}[15]$ ) were considered and are summarized in Table 1 [16]. These dimensions are suitable for water and sanitary applications as well as stormwater drainage. The level to be detected has been characterised as "Empty" and "Full" for the pipe without and with water, respectively. The first-order Debye model with $\varepsilon_{\text {static }}=78.4$, $\varepsilon_{\infty}=3.1$, relaxation time $\tau=8.27 \mathrm{ps}$, and $\mu=0.99$ has been used to model the dispersive behaviour of water.

2.3. Excitation Signal and Fidelity Factor Measurement. Choosing the excitation signal for the antenna is a very critical aspect of this analysis as the device is designed to operate as both sensor and transmitter. Several design factors need to be considered to select a suitable signal to feed the sensor/antenna:

(i) antenna dimensions are inversely proportional to the lower edge frequency;

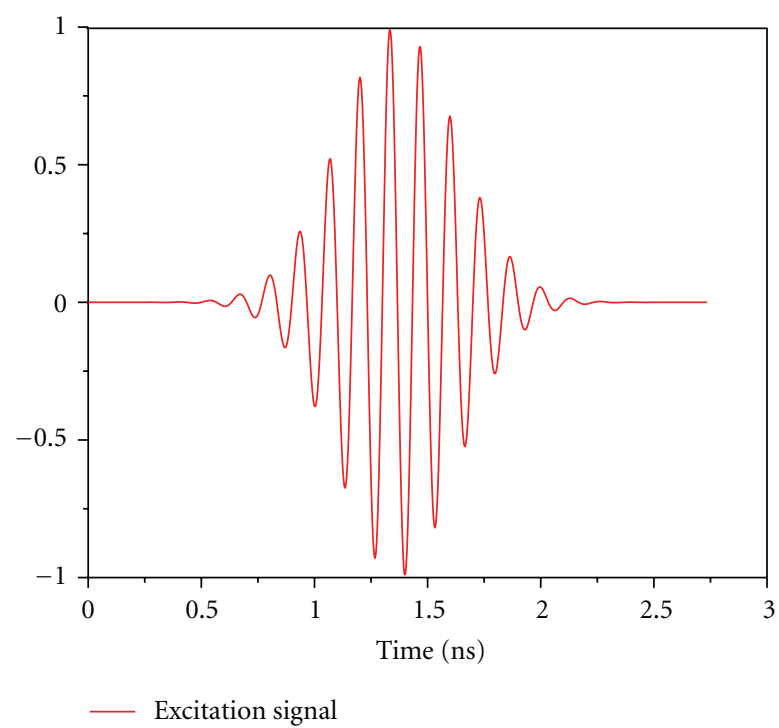

FIgURE 4: Normalised input signal.

(ii) considering that the antenna is placed close to water, the lower edge frequency must be selected in such a way this remains efficient in the lower part of the operating bandwidth;

(iii) the system sensitivity to water loading is dependent on the bandwidth over which the fidelity factor is measured.

In Figure 4 the normalised input signal selected for this application is plotted in the time domain. A convenient compromise between antenna efficiency and suitable fidelity factor sensitivity to the testing environment can be reached by using a Gaussian pulse centred at $7.5 \mathrm{GHz}$. Its frequency spectrum $20 \mathrm{~dB}$ bandwidth spans between 6.2 and $8.8 \mathrm{GHz}$.

\section{Frequency Domain Performance}

In this section the antenna performance is evaluated in the frequency domain when it is mounted conformally on the pipe configurations given in Table 1. Different diameters of curvature and thickness of the PVC pipes affect the antenna behaviour both in terms of impedance matching and radiation properties. But the presence of water inside the pipe generates the most evident variations. Figure 5 shows the resilience of the antenna impedance matching properties for the different pipe configurations when empty or full of water. It can be observed that the return loss curve drops to lower values when the pipe is filled with water as this introduces more losses. This effect is more evident for configurations $A$ and $C$ due to the thinner PVC layer between the antenna and the water content.

As from simulations the antenna shows stable radiation properties in terms of pattern shape over the operating bandwidth $(6.2-8.8 \mathrm{GHz})$, the $H$-plane diagrams are only displayed at the centre frequency $7.5 \mathrm{GHz}$ (Figure 6). A significant gain drop occurs when the pipe is filled with water due to increased losses and dielectric loading. However, for 


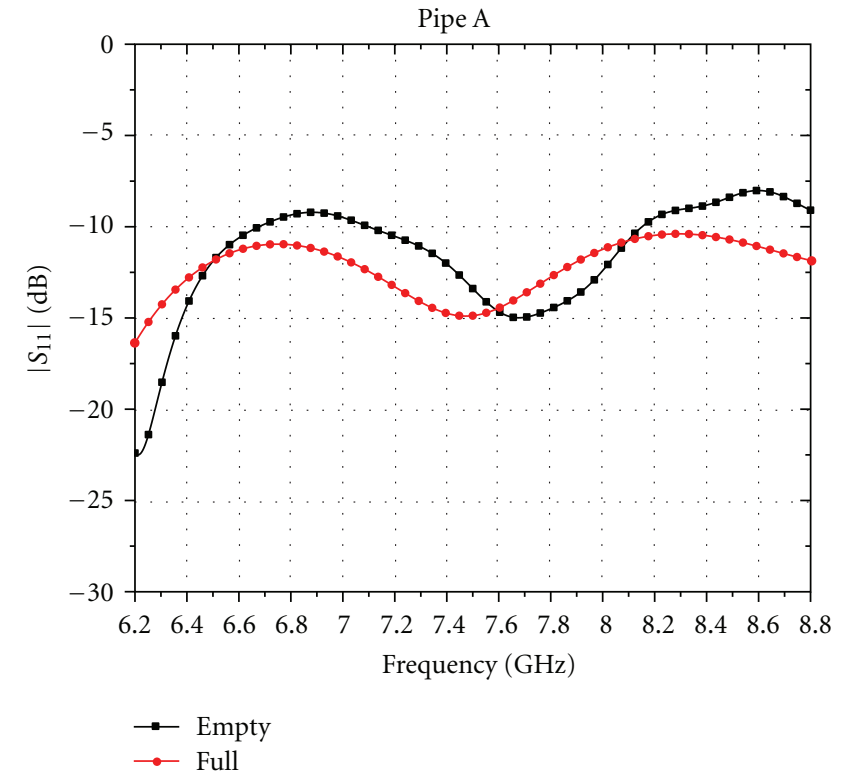

(a)

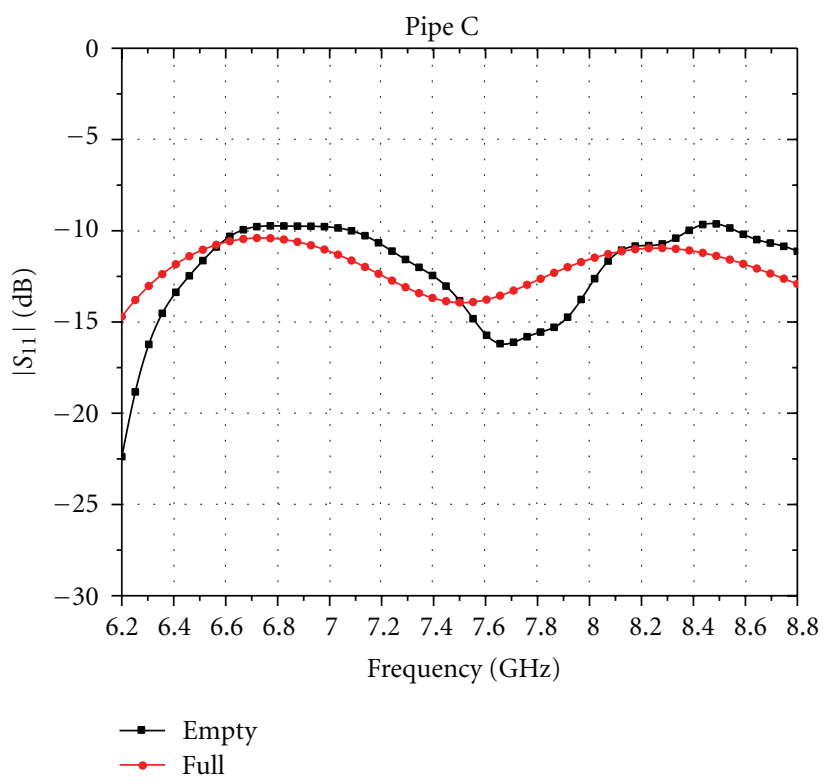

(c)

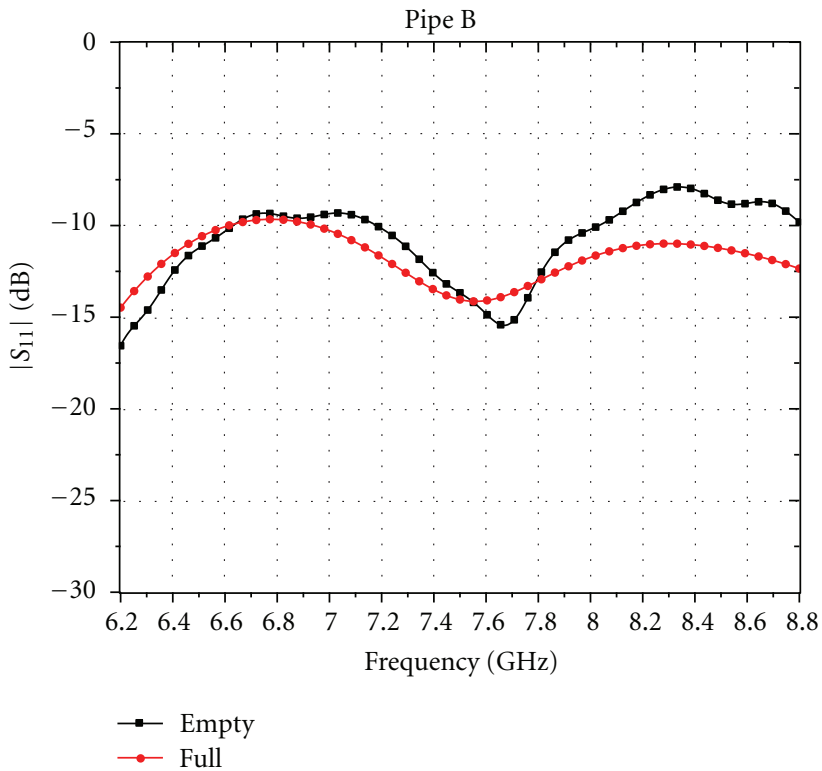

(b)

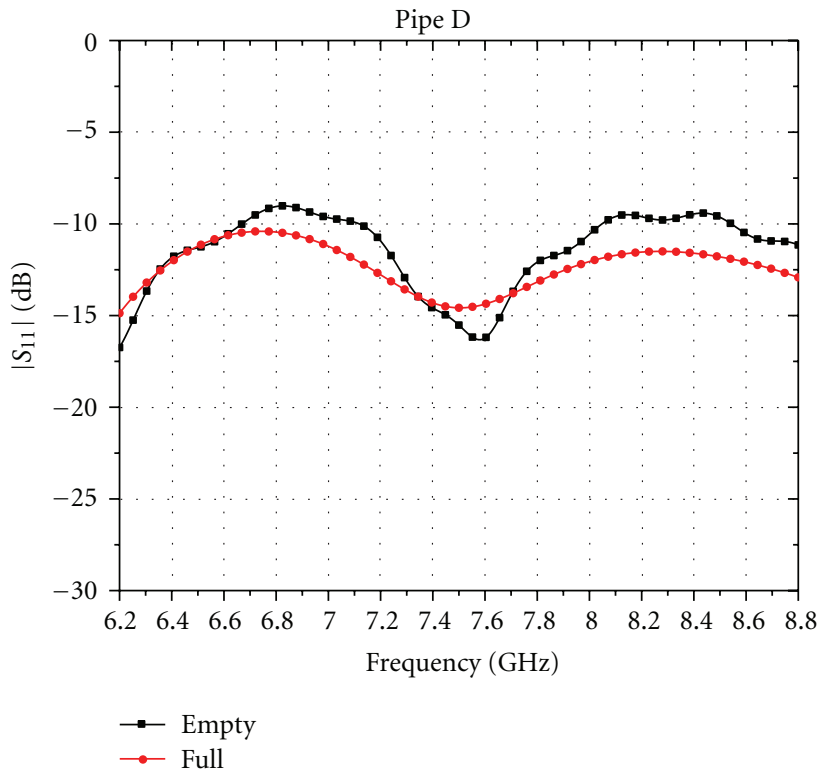

(d)

FIGURE 5: $S_{11}$ comparison of antenna conformal to pipe configurations (a)-(d) without water (black trace) and with water (red trace).

all configurations investigated the antenna radiation remains efficient with and without water inside the pipe. Also the direction of maximum radiation changes because of the reflecting behaviour of the water content. In fact, this shifts towards the angular sector $30^{\circ}-50^{\circ}$ when the pipe is full of water.

\section{Time Domain Performance}

As the antenna is required to provide simultaneous transmission and sensor function, an angular sector where good radiation efficiency and sensing characteristics needs to be identified. As shown in Figure 6, for all the configurations investigated, a satisfactory compromise between the gain performance measured in the $H$-plane with and without water is achieved in the range $60^{\circ} \leq \theta \leq 70^{\circ}$. Moreover, in this spatial region the fidelity factor assumes values above $90 \%$ that corresponds to a reliable radio link for pulse communications in all the analysed scenarios. Outside the interval $60^{\circ}-70^{\circ}$ a combined suitable performance in terms of gain (above $0 \mathrm{dBi}$ ) and FF (above $80 \%$ ) was not found for all the scenarios under investigation. On the other hand, the 

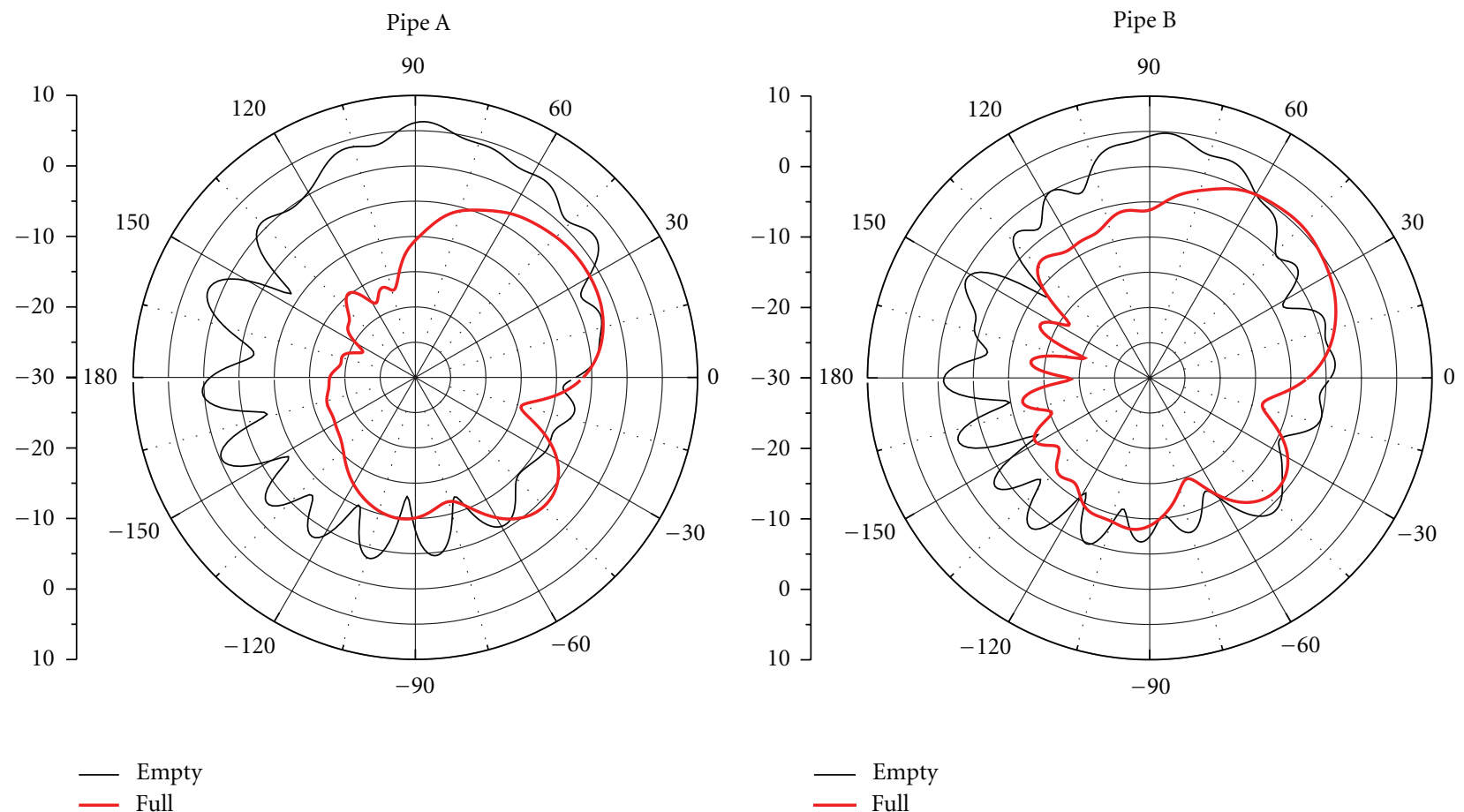

(a)

(b)
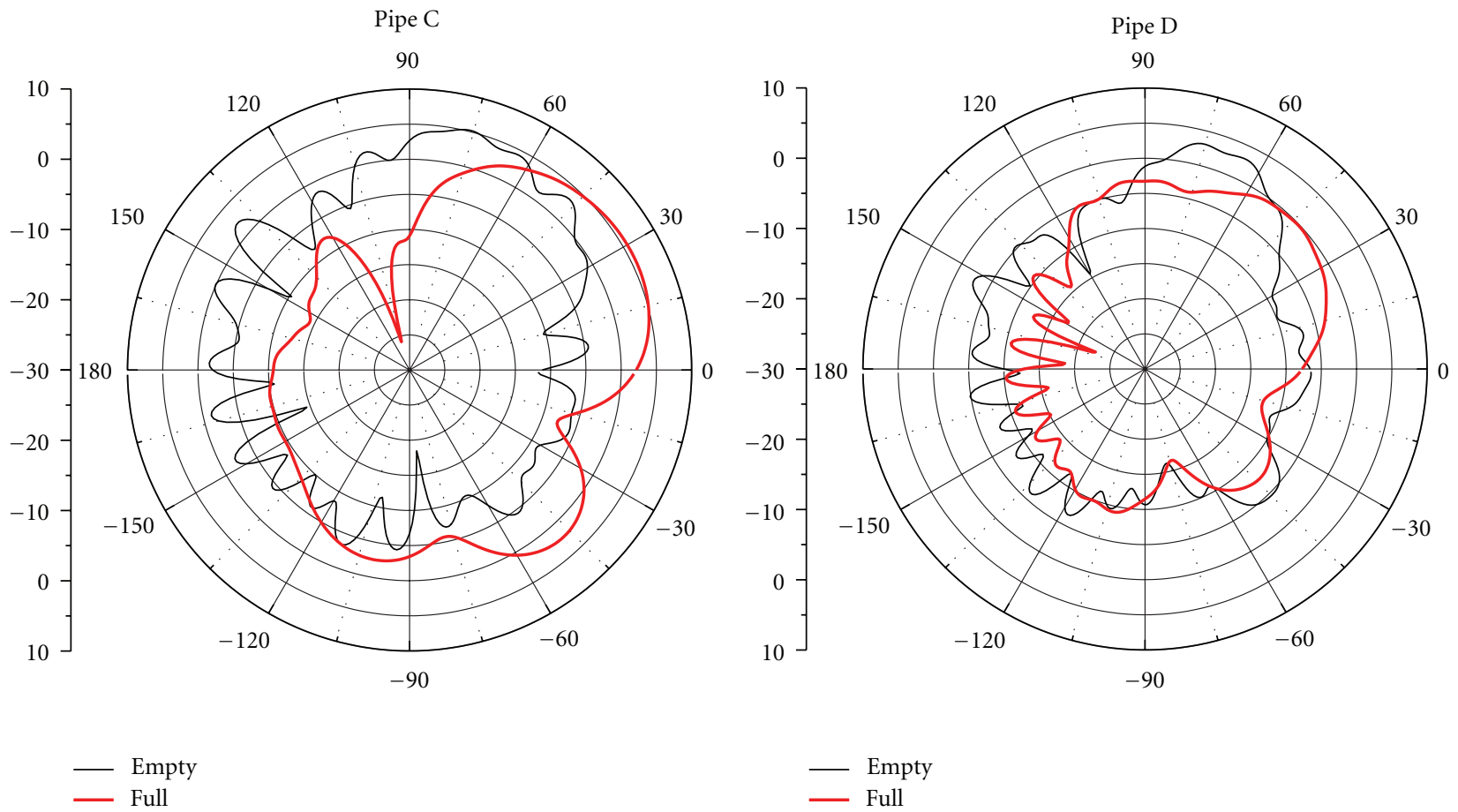

(c)

(d)

FiguRE 6: $H$-Plane radiation pattern comparison at $7.5 \mathrm{GHz}$ of antenna conformal to pipe configurations (a)-(d) without water (black trace) and with water (red trace). 


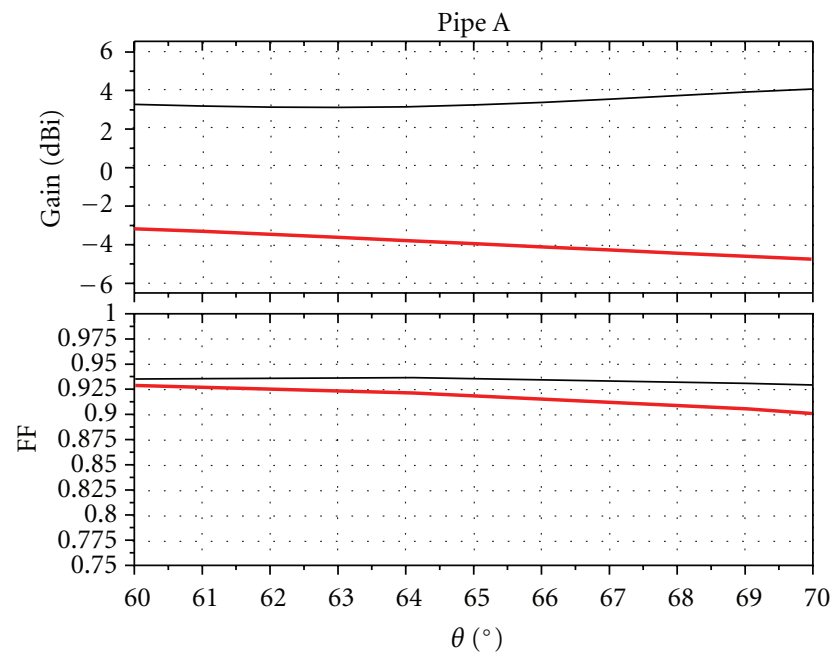

(a)

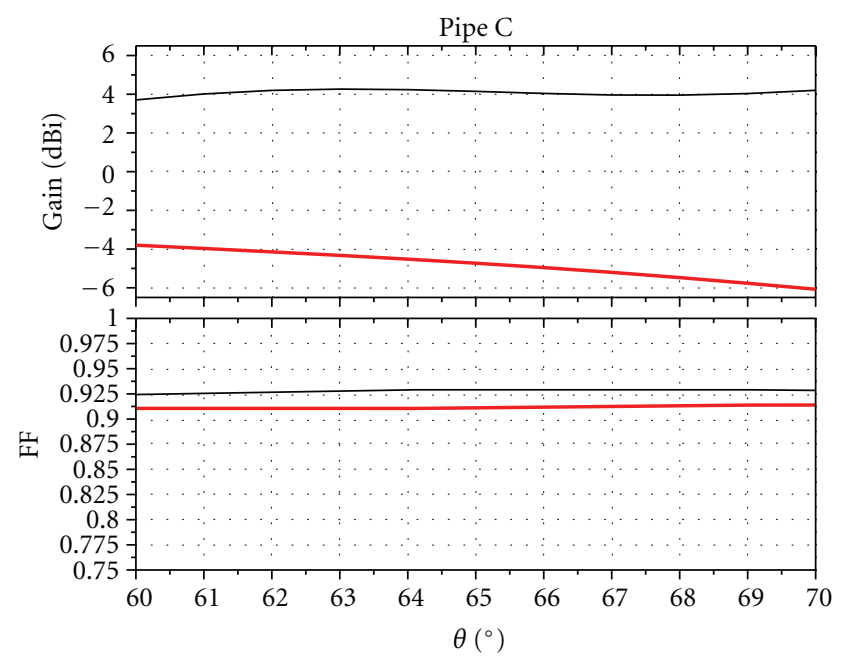

(c)

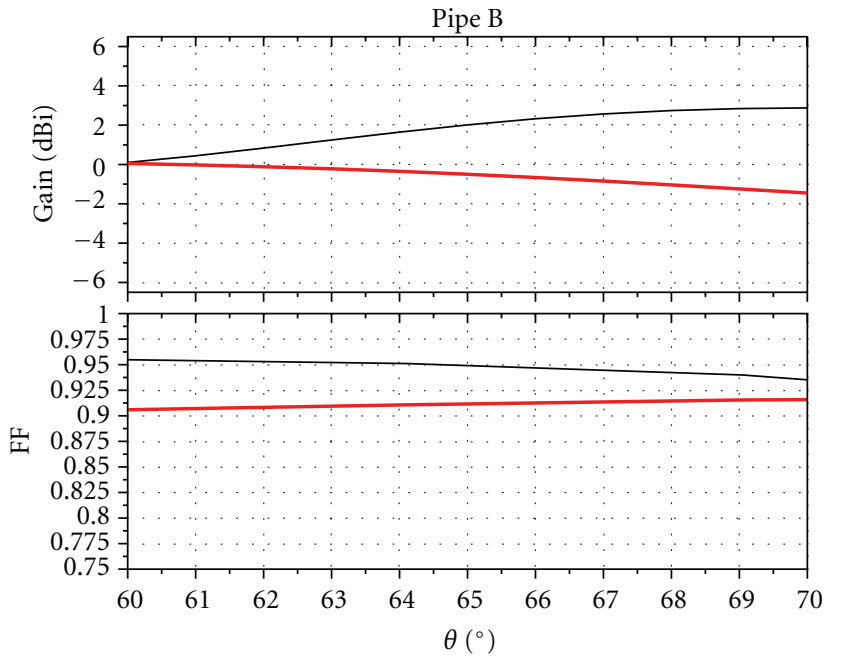

(b)

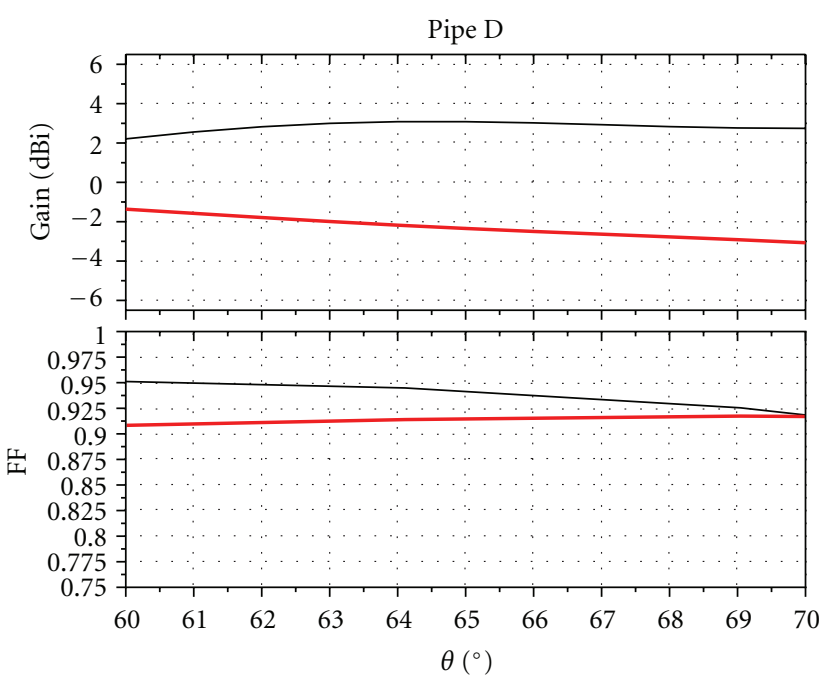

(d)

FIGURE 7: Realised gain and fidelity factor comparison at $7.5 \mathrm{GHz}$ in the range $60^{\circ} \leq \theta \leq 70^{\circ}$ of antenna conformal to pipe configurations (a)-(d) without water (black trace) and with water (red trace).

fidelity factor can be also used as a valid figure of merit for sensing purposes. By definition, it depends on the variations of the transmitted signal across the entire bandwidth. As the antenna transmitting properties change when water fills the pipe, this effect is promptly expressed as a fidelity factor distortion. Figure 7 shows fidelity factor variations in the order of $6 \%$ between the cases "Empty" and "Full." Once the system is properly characterised, those deviations can be used to monitor the water content inside the PVC pipes and simultaneously transmit the information to an external metering station.

\section{Conclusions}

A UWB directional antipodal Vivaldi antenna has been designed for water pipe sensing and telemetry. While the antenna impedance remains matched when placed on the four pipes investigated, gain and fidelity factor undergo a notable reduction with the pipe filled of water. The dispersive property of the pipe material and the water will modify the transfer function of the antenna, distorting the radiated and received signal shape. However, by focusing on the bandwidth between 6.2 and $8.8 \mathrm{GHz}$ and the angular range $60^{\circ} \leq \theta \leq 70^{\circ}$ in the $\mathrm{H}$-plane, the antenna radiates efficiently in all the scenarios investigated in terms of gain and fidelity factor figures. These properties suggested using the same antenna for simultaneous sensing and telemetry applications. As the water content causes fidelity factor variations between 90 and $96 \%$, this figure is proposed as a reliable sensing parameter to assess the pipe content status. At the same time, as values above $80 \%$ secure good radiolinks for impulse communications, the antenna is able to transmit real-time information to an external telemetry station. 


\section{References}

[1] O. Duran, K. Althoefer, and L. D. Seneviratne, "State of the art in sensor technologies for sewer inspection," IEEE Sensors Journal, vol. 2, no. 2, pp. 73-81, 2002.

[2] S. K. Sinha, S. R. Iyer, and M. C. Bhardwaj, "Non-contact ultrasonic sensor and state-of-the-art camera for automated pipe inspection," in Proceedings of the 2nd International Conference on Sensors, pp. 493-498, October 2003.

[3] F. Gomez, K. Althoefer, and L. D. Seneviratne, "Modeling of ultrasound sensor for pipe inspection," in Proceedings of the IEEE International Conference on Robotics and Automation, pp. 2555-2560, September 2003.

[4] O. Hunaidi, "Ground penetrating radar for detection of leaks in buried plastic water distribution pipes," in Proceedings of the 7th International Conference on Ground Penetrating Radar (GPR'98), pp. 783-786, Lawrence, Kan, USA, 1998.

[5] S.-Y. Hyum, Y.-S. Jo, H.-C. Oh, and S.-Y. Kim, "An experimental study on a ground penetrating radar for detecting waterleaks in buried water transfer pipes," in Proceedings of the 6th International Symposium on Antennas, Propagation and EM Theory, pp. 597-599, 2003.

[6] J. H. Goh, A. Shaw, J. D. Cullen et al., "Water pipe leak detection using electromagnetic wave sensor for the water industry," in Proceedings of the IEEE Symposium on Computers \& Informatics (ISCI '11), pp. 290-295, Kuala Lumpur, Malaysia.

[7] D. Gaetano, M. J. Ammann, P. McEvoy, M. John, L. Keating, and F. Horgan, "A conformal UWB directional antenna," in Proceedings of the 5th European Conference on Antennas and Propagation (EUCAP'11), pp. 1113-1116, April 2011.

[8] A. Kadri, A. Abu-Dayya, D. Trinchero, and R. Stefanelli, "Design of radio-acoustic sensors for leakage detection in underground water pipes," in Proceedings of the 12th International Conference on Electromagnetics in Advanced Applications (ICEAA'10), pp. 867-870, September 2010.

[9] A. Dumoulin, M. John, M. J. Ammann, and P. McEvoy, "Optimized monopole and dipole antennas for UWB asset tag location systems," IEEE Transactions on Antennas and Propagation, vol. 60, no. 6, Article ID 6192292, pp. 2896-2904, 2012.

[10] On-line, http://www.cst.com/.

[11] D. Lamensdorf and L. Susman, "Baseband-pulse-antenna techniques," IEEE Antennas and Propagation Magazine, vol. 36, no. 1, pp. 20-30, 1994.

[12] D. Ghosh, A. De, M. C. Taylor, T. K. Sarkar, M. C. Wicks, and E. L. Mokole, "Transmission and reception by ultra-wideband (UWB) antennas," IEEE Antennas and Propagation Magazine, vol. 48, no. 5, pp. 67-99, 2006.

[13] E. Gazit, "Improved design of the vivaldi antenna," IEE Proceedings Microwaves, Antennas and Propagation, vol. 135, no. 2, pp. 89-92, 1988.

[14] K. C. Gupta, R. Garp, I. Bahl, and P. Bhartia, Microstrip Lines and Slotlines, Artech House, Dedham, Mass, USA, 2nd edition, 1979.

[15] M. Adous, P. Quéffélec, and L. Laguerre, "Coaxial/cylindrical transition line for broadband permittivity measurement of civil engineering materials," Measurement Science and Technology, vol. 17, no. 8, article no. 026, pp. 2241-2246, 2006.

[16] ASTM Standard D1785, "ASTM D1785-06 Standard Specification for Polyvinyl Chloride (PVC) plastic pipe, schedules 40, 80, and 120," ASTM International, West Conshohocken, Pa, USA, 2006, http://www.astm.org/. 

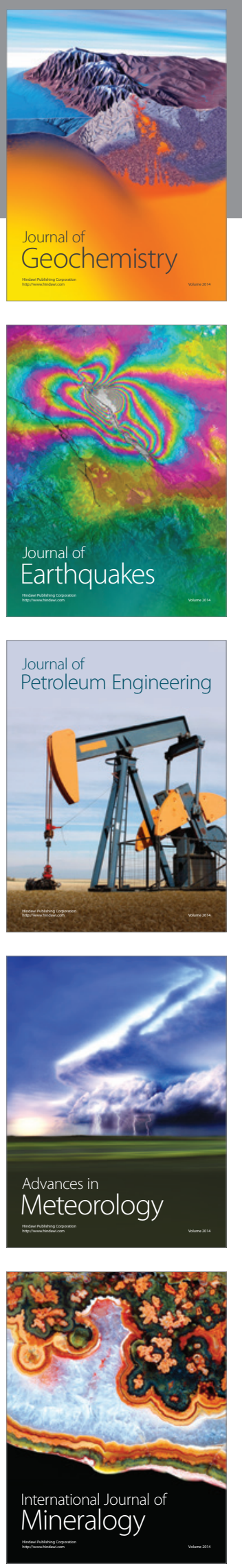
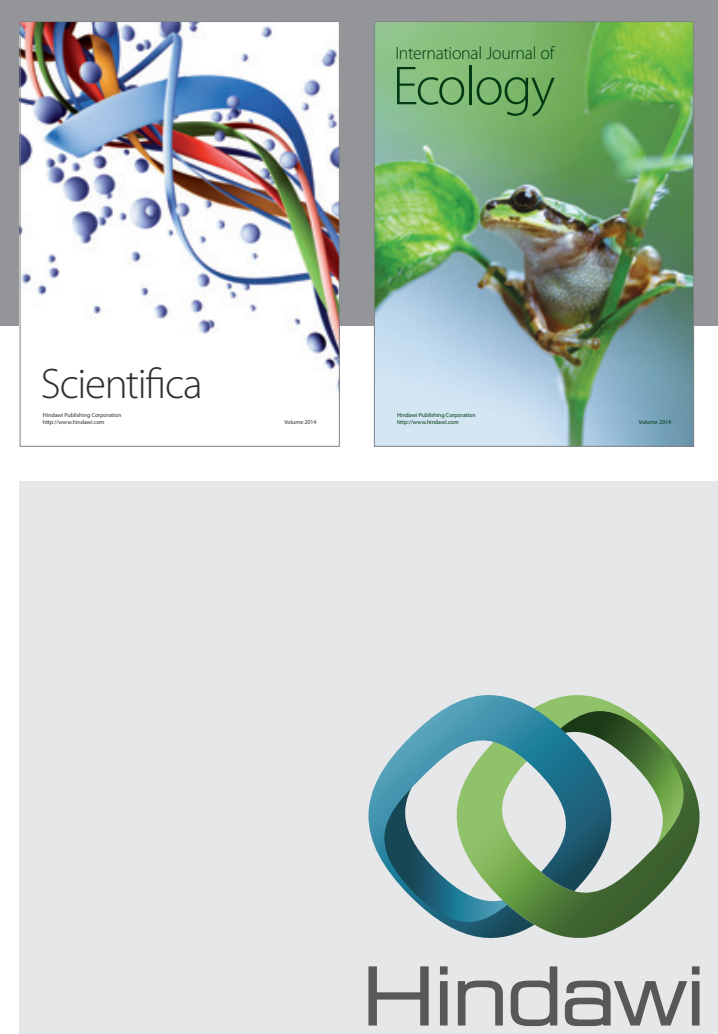

Submit your manuscripts at http://www.hindawi.com
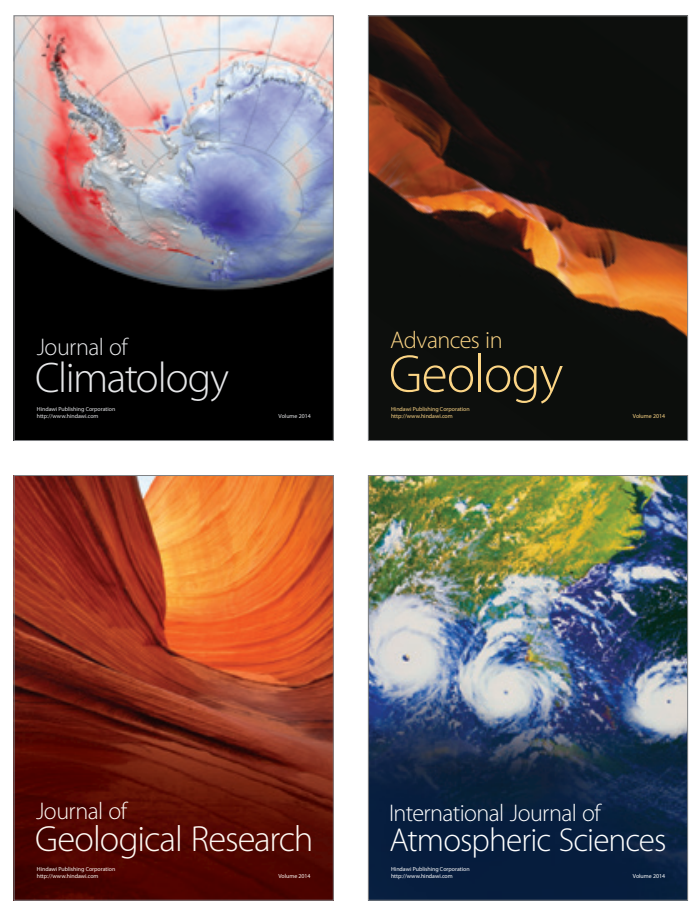
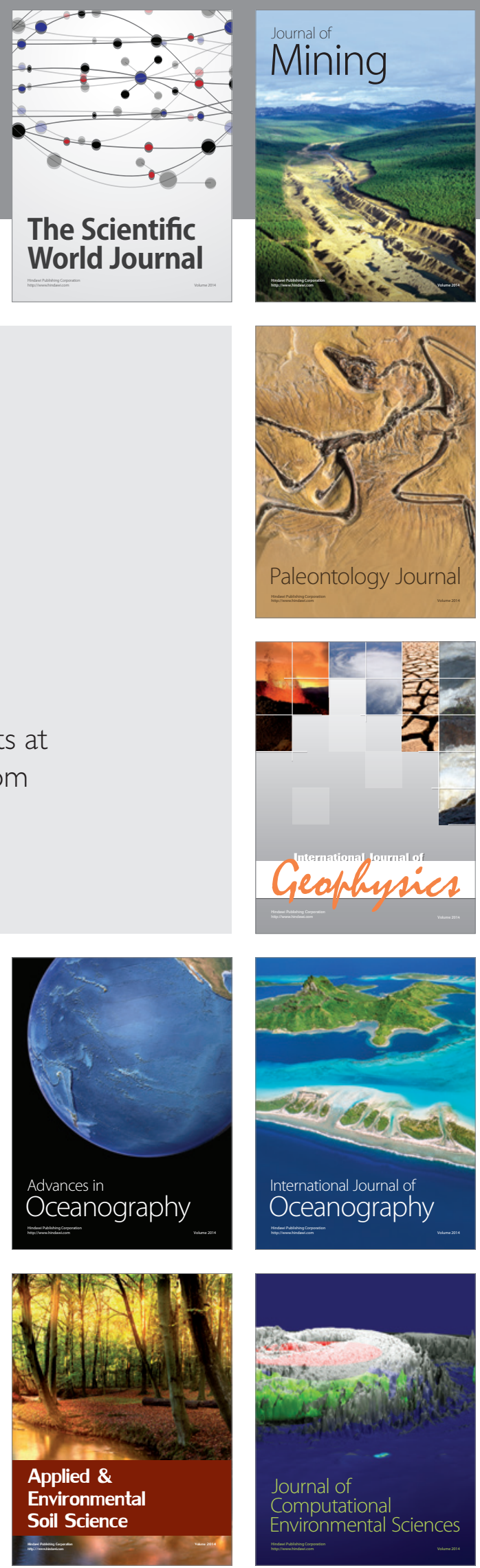